\section{Le baclofène est un modulateur allostérique du récepteur CXCR4}

Alice Guyon
Institut de pharmacologie moléculaire et cellulaire (IPMC), UMR 7275 CNRS/Université Nice Sophia Antipolis, 660 , route des Lucioles, ZA Sophia Antipolis, 06560 Valbonne, France.

alice.guyon@ipmc.cnrs.fr
> Le baclofène, commercialisé à l'origine sous le nom de Liorésal ${ }^{\circledR}$, est un dérivé aromatique halogéné de l'acide gamma-aminobutyrique (GABA). C'est un myorelaxant inhibant les réflexes mono- et polysynaptiques au niveau de la moelle épinière, dont l'effet se concentre sur les muscles squelettiques [1]. Le baclofène a beaucoup fait parler de lui récemment car sa prescription vient d'être élargie en France à une utilisa- tion dans le sevrage alcoolique [2, 3]. On attribue ses effets à son action agoniste de certains récepteurs au $G A B A$, les récepteurs $G A B A_{B}$, qui sont des récepteurs couplés aux protéines $G$ largement exprimés dans le système nerveux $[4,5]$.

Plusieurs observations nous ont conduits à penser que l'action du baclofène sur son récepteur $G_{B B A_{B}}$ ne suffisait pas à expliquer tous ses effets. $\varepsilon n$ effet, nous montrons dans un article paru récemment dans The Journal of Neuroscience [6] que, outre son action bien connue sur les récepteurs $G_{B B}$, le baclofène agit également de manière allostérique en bloquant un autre récepteur transmembranaire couplé aux protéines $G$, le récepteur CXCR4 de la chimiokine CXCL12/SDFl $\alpha$ (stromal cell-derived factor).

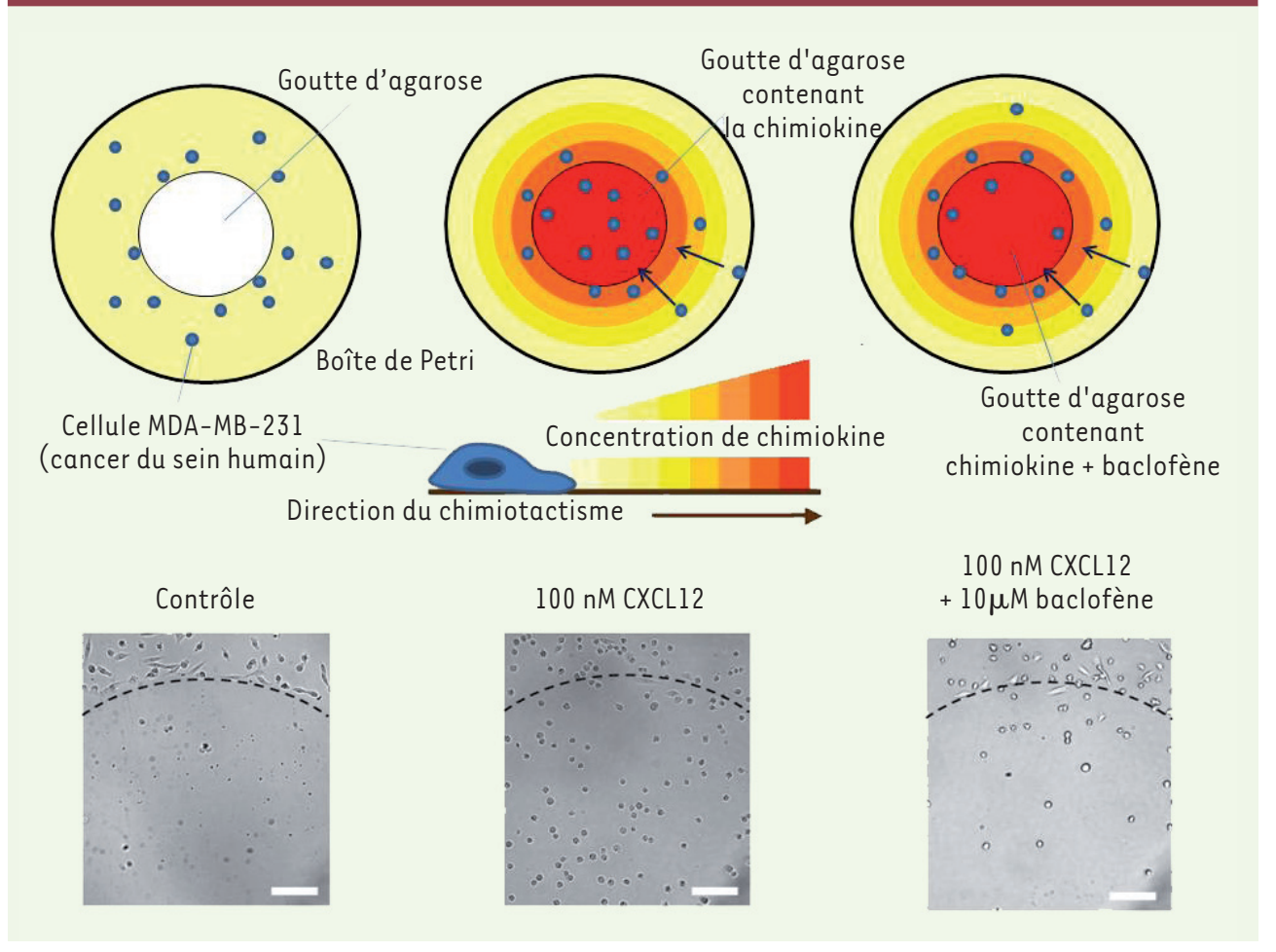

Figure 1. Quantification des effets du baclofène sur le chimiotactisme induit par CXCL12 sur des cellules de cancer du sein humain (de la lignée MDA-MB-231). Une goutte d'environ $1 \mathrm{~mm}$ de diamètre, préparée à partir d'une solution d'agarose, est déposée au fond d'une boîte de Petri de $35 \mathrm{~mm}$ de diamètre, puis la solution contenant les cellules de la lignée MDA-MB-231 est ajoutée et la boîte est mise à incuber durant la nuit. Lorsque la goutte est préparée à partir d'une solution d'agarose ne contenant pas la chimiokine, les cellules restent en bordure de la goutte (à gauche) alors que lorsque la goutte est faite à partir d'une solution d'agarose contenant $100 \mathrm{nM}$

de CXCL12 (milieu), les cellules de la lignée MDA-MB-231 rampent sous la goutte, attirées par la chimiokine. Lorsque la goutte est préparée à partir d'une solution d'agarose contenant à la fois $100 \mathrm{nM}$ de CXCL12 et $10 \mu \mathrm{M}$ de baclofène (à droite), le chimiotactisme est largement inhibé et très peu de cellules parviennent à ramper sous la goutte. La quantification se fait en comptant le nombre de cellules ayant pénétré sous la goutte sur des photos prises au microscope telles que celles présentées en dessous des schémas. Barre d'échelle : $100 \mu$ m. Les pointillés représentent la limite de la goutte. 


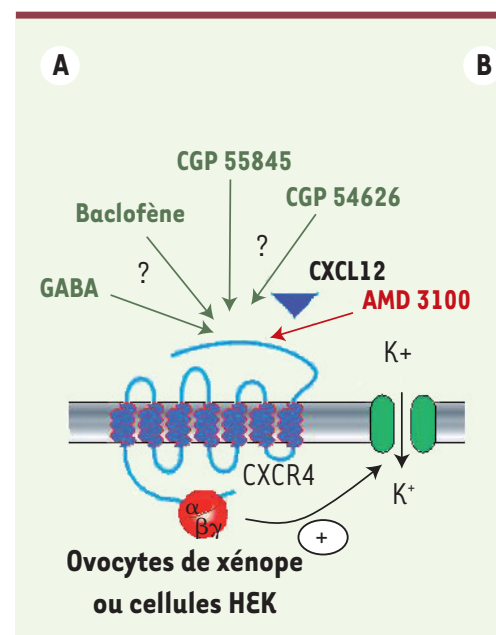

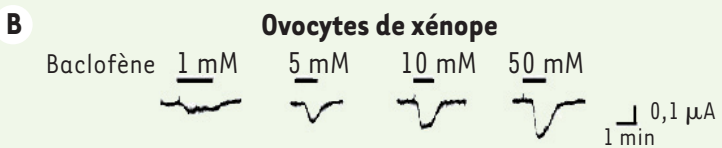

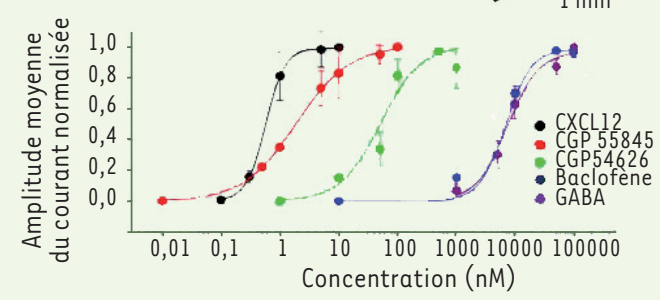

C

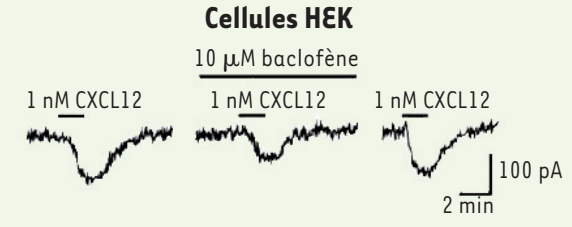

Figure 2. Étude électrophysiologique des effets du baclofène sur le CXCR4. A. Coexpression du récepteur CXCR4 avec un canal rapporteur, le canal potassique de type GIRK, à la membrane d'ovocytes de xénope et de cellules HEK (human embryonnic kidney) afin de tester les effets des agonistes et antagonistes des

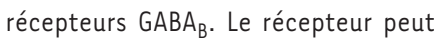
être activé par son ligand, le CXCL12, ce qui conduit à l'activation d'une protéine $G$ et à l'activation du canal GIRK (canal potassique activé par les protéines $\mathrm{G}$ ). Le récepteur CXCR4 peut être bloqué de manière compétitive par un antagoniste, I'AMD 3100.

B. L'application de concentrations

croissantes de baclofène sur des ovocytes de xénope coexprimant le CXCR4 et le GIRK induit des courants potassiques d'amplitude croissante, montrant que dans ces cellules, le baclofène se comporte comme un agoniste du CXCR4. En haut sont présentés des exemples d'enregistrements, en bas les courbes concentrations-réponses obtenues à partir d'enregistrements similaires pour le baclofène (en bleu) et pour d'autres agonistesantagonistes des récepteurs $G_{A B A}$. C. L'application de CXCL12 sur des cellules HEK coexprimant le CXCR4 et le GIRK induit des courants potassiques qui sont partiellement bloqués de manière réversible par le baclofène.

\section{Le baclofène modifie les réponses du récepteur CXCR4}

Les chimiokines sont des substances chimioattractantes qui jouent un rôle important dans le système immunitaire et dans le cancer, mais exercent aussi leurs actions dans le système nerveux $[7,8]$. Parmi les chimiokines, CXCL12/ SDFl $\alpha$ a beaucoup attiré l'attention notamment parce que l'un de ses deux récepteurs, le CXCR4, est également un des corécepteurs du VIH, le virus de l'immunodéficience humaine [9].

Une étude [10] avait montré que des agonistes et des antagonistes du récepteur $G_{A B A_{B}}$ peuvent empêcher les effets chimioattractants du SDFl $\alpha$ sur des cellules cancéreuses exprimant spontanément le récepteur CXCR4, ce que nous avons confirmé [6] (Figure 1). De même, nous avons observé fortuitement qu'un antagoniste des récepteurs $G A B A_{B}$, le CGP 55845A, inhibe complètement les actions de la chimiokine sur les courants calciques membranaires de certains neurones du cerveau, effets connus pour être dus à la stimulation du CXCR4 [11].
Nous avons d'abord pensé que les récepteurs $G A B A_{B}$ et CXCR4 pouvaient interagir dans la membrane des cellules: en effet, on sait que CXCR4 peut former des hétérodimères avec d'autres récepteurs couplés aux protéines $G[12,13]$. Toutefois, en collaboration avec une équipe américaine $^{1}$ nous avons fait exprimer à la membrane d'ovocytes de xénope les deux récepteurs marqués, I'un par un composé fluorescent émettant dans le rouge, l'autre par un composé fluorescent émettant dans le vert; une analyse par microscopie TIRF (total internal reflexion fluorescence) nous a permis de constater que les deux types de récepteurs ne sont pas colocalisés dans la membrane.

Toujours dans ce système, nous avons ensuite fait exprimer à la membrane le CXCR4 avec un canal ionique rapporteur, le canal potassique activé par les protéines $\mathrm{G}$ appelé GIRK ( $G$-protein

\footnotetext{
${ }^{1}$ Le laboratoire de Ehud Isacoff, Department of Molecular and Cellular Biology and Helen Wills Neuroscience Institute, University of California, Berkeley, Berkeley, CA 94720 , États-Unis.
}

activated inward rectifier $K^{+}$channel) (Figure 2). Quand le CXCR4 est activé par son ligand CXCL12/SDFl $\alpha$, I'activation du récepteur ouvre le canal GIRK, ce qui se traduit par un courant mesurable à la membrane par des techniques d'électrophysiologie (Figure 2A). Nous avons caractérisé l'interaction des agonistes/antagonistes des récepteurs $G_{A B A_{B}}$ avec le CXCR4 et montré que ces substances modifient l'effet du SDFl $\alpha$ sur son récepteur par une action allostérique. De manière intéressante, dans les ovocytes de xénope, les substances agonistes/antagonistes des récepteurs $G_{B B A}$ se comportent comme des agonistes du CXCR4 (Figure 2B). Ce résultat contredit nos observations initiales d'un blocage de CXCR4 par ces substances. À l'aide de la technique TIRF, nous avons montré que les récepteurs CXCR4 ne forment pas de dimères lorsqu'ils sont exprimés à la membrane des ovocytes de xénope, contrairement à ce qui est décrit dans les cellules de mammifères $[14,15]$. Nous avons alors utilisé un autre système cellulaire, les cellules 


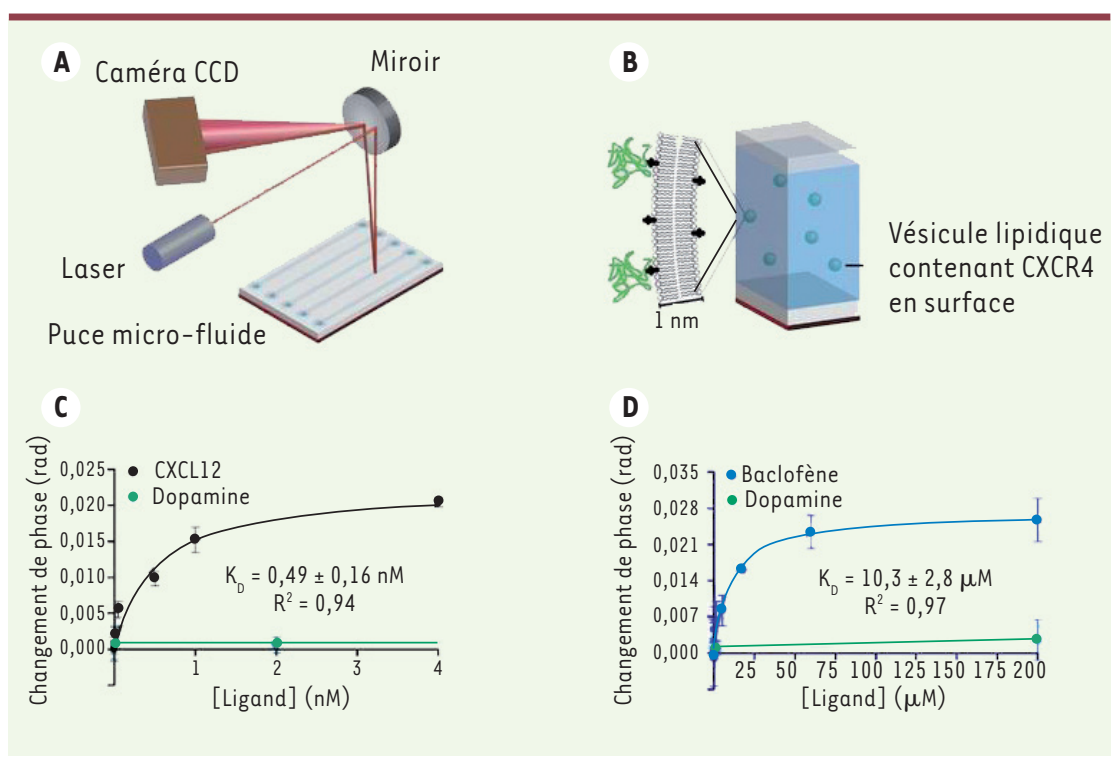

Figure 3. Vérification d'une interaction directe entre CXCR4 et baclofène par la technique d'interférométrie par diffusion rétrograde de la lumière. $A$. Dispositif expérimental permettant de réaliser des mesures d'interférométrie par diffusion rétrograde de la lumière. Dans chaque puits de la puce est disposé un liquide contenant des vésicules lipidiques en suspension exprimant le CXCR4 à leur surface et différentes concentrations des ligands à tester. Le changement de phase induit par la liaison du ligand sur son récepteur est mesuré grâce à la caméra (adapté de [16]). B. Schéma représentant les vésicules formées de bicouches lipidiques contenant exclusivement la protéine CXCR4 à leur surface. C. Courbe de changement de phase en fonction de la concentration de ligand obtenue pour CXCL12 (le ligand du CXCR4). Le $K_{D}$ obtenu est conforme à celui obtenu avec d'autres techniques. La dopamine est utilisée comme contrôle négatif. $\boldsymbol{D}$. Courbe de changement de phase en fonction de la concentration de ligand obtenue pour le baclofène, démontrant que le baclofène se lie directement au CXCR4 avec une affinité similaire à celle avec laquelle il se lie au récepteur $\mathrm{GABA}_{B}$ (la dopamine est utilisée comme contrôle négatif).

HEK, et exprimé les récepteurs CXCR4 à la membrane de ces cellules humaines. Dans ces conditions, les agonistes/ antagonistes des récepteurs $G_{A B A}$ se comportent effectivement comme des bloqueurs allostériques du récepteur.

\section{Le baclofène se lie directement au CXCR4}

En collaboration avec une autre équipe américaine ${ }^{2}$, nous avons pu confirmer l'existence d'une interaction directe entre ces agonistes/antagonistes des récepteurs $G_{A B A_{B}}$ et le CXCR4 en exprimant ce récepteur dans des bicouches lipidiques et en utilisant la technique d'interfé-

\footnotetext{
${ }^{2}$ Le laboratoire de Darryl Bornop, Vanderbilt University/ Department of Chemistry, 7300 Stevenson Center, Nashville, Tennessee 37235-1822, États-Unis.
}

CXCL12 pour son récepteur était conforme à celui attendu et qu'un neurotransmetteur contrôle ne se liant pas au récepteur - la dopamine - n'induisait pas de changement de phase (Figure $3 \mathrm{C}$ ). Nous avons ensuite pu montrer que le baclofène (Figure 3D) se lie au CXCR4 avec une affinité similaire à son affinité pour le récepteur $G A B A_{B}$. Il en a été de même pour le GABA lui-même et deux antagonistes connus des récepteurs $G A B A_{B}[6]$. Le site d'action précis sur la molécule reste à déterminer et des études en cristallographie sont en cours, mais nous avons pu montrer par la technique de FRET (fluorescence resonance energy transfer) [6] qu'il s'agit d'un site relativement distant du site de liaison de la chimiokine sur son récepteur.

\section{En conclusion}

Nous montrons que le baclofène, outre ses effets bien connus sur les récepteurs $G A B A_{B}$, exerce aussi un effet allostérique sur les récepteurs CXCR4 qui pourrait participer à ses mécanismes d'action. Dans la mesure où certains neurones expriment CXCR4 au niveau de synapses GABAergiques, ce travail ouvre de nouvelles perspectives sur l'implication des effets antagonistes du baclofène et $d u$ GABA lui-même sur le CXCR4 au niveau du système nerveux. Cette étude ouvre également de nouvelles perspectives thérapeutiques dans le traitement de certains cancers; elle incite à rester vigilant sur les effets putatifs du baclofène sur le système immunitaire des patients traités, en particulier ceux atteints de cancer ou d'infection par le VIH. $\diamond$

Baclofen and other GABA $A_{B}$ receptor agents are allosteric modulators of the CXCL12 chemokine receptor CXCR4

\section{REMERCIEMENTS}

L'auteur remercie Franck Aguila pour ses conseils artistiques sur les figures, Thomas Lorivel et Joëlle Chabry pour leur relecture attentive, Audrey Recouly, Fabienne Chevallier et Florence Servent de la Société CISBIO Bioassays, Codolet, France pour les expériences en FRET utilisant la technologie 
Tag-lite et Richard Miller (Chicago) pour nous avoir fourni le clone de CXCR4.

\section{LIENS D'INTÉRÊT}

L'auteur déclare n'avoir aucun lien d'intérêt concernant les données publiées dans cet article.

\section{RÉFÉRENCES}

1. Rekand T, Gronning M. Treatment of spasticity related to multiple sclerosis with intrathecal baclofen: a long-term follow-up. J Rehabil Med 2011 ; 43 : 511-4.

2. Gorsane MA, Kebir 0, Hache G, et al. Is baclofen a revolutionary medication in alcohol addiction management? Review and recent updates. Subst Abus 2012 ; 33: 336-49.

3. Addolorato G, Leggio L, Agabio R, et al. Baclofen: a new drug for the treatment of alcohol dependence. Int J Clin Pract 2006 ; 60 : 1003-8.

4. Bowery NG. GABAB receptor pharmacology. Annu Rev
Pharmacol Toxicol 1993 ; 33 : 109-47.

5. Froestl, W. Chemistry and pharmacology of GABAB receptor ligands. Adv Pharmacol $2010 ; 58$ : 19-62.

6. Guyon A, Kussrow A, Olmsted IR, et al. Baclofen and other $G A B A B$ receptor agents are allosteric modulators of the CXCL12 chemokine receptor CXCR4. J Neurosci 2013 ; 33 : 11643-54.

7. Banisadr G, Rostene W, Kitabgi P, Parsadaniantz S. Chemokines and brain functions. Curr Drug Targets Inflamm Allergy $2005 ; 4$ : 387-99.

8. Guyon A, Massa F, Rovere C, Nahon JL. How cytokines can influence the brain: a role for chemokines? J Neuroimmunol 2008 ; $198:$ 46-55.

9. Toth PT, Ren D, Miller RJ. Regulation of CXCR4 recepto dimerization by the chemokine SDF-lalpha and the HIV-1 coat protein gp 120: a fluorescence resonance energy transfer (FRET) study. J Pharmacol Exp Ther $2004 ; 310: 8-17$.

10. Ortega A. A new role for GABA: inhibition of tumor cell migration. Trends Pharmacol Sci 2003 ; 24 : 151-4.

11. Guyon A, Skrzydelski D, Rovère $C$, et al. Stromalcell-derived factor lalpha /CXCL12 modulates high- threshold calcium currents in rat substantia nigra. Eur J Neurosci $2008 ; 28: 862-70$.

12. Pello OM, Martínez-Muñoz L, Parrillas V, et al. Ligand stabilization of CXCR4/delta-opioid receptor heterodimers reveals a mechanism for immune response regulation. Eur J Immunol 2008 ; 38 : 537-49.

13. Sohy D, Parmentier M, Springael Jy. Allosteric transinhibition by specific antagonists in CCR2/CXCR4 heterodimers. J Biol Chem 2007 ; 282 : 30062-9.

14. Percherancier $Y$, Berchiche $Y A$, Slight I, et al. Bioluminescence resonance energy transfer reveals ligand-induced conformational changes in CXCR4 homo- and heterodimers. J Biol Chem 2005; 280 : 9895-903.

15. Babcock GJ, Farzan M, Sodroski J. Ligand-independent dimerization of CXCR4, a principal HIV-1 coreceptor. J Biol Chem $2003 ; 278: 3378-85$.

16. Baksh MM, Kussrow AK, Mileni M, et al. Label-free quantification of membrane-ligand interactions using backscattering interferometry. Nat Biotechnol 2011 ; $29: 357-60$.

\section{NOUVELLE}

\section{Contrôle de la mitophagie par les microARN \\ Une étape clé de l'érythropoïèse}

Isabelle Barde ${ }^{1}$, Benjamin Rauwel ${ }^{1}$, Ray Marcel Marin-Florez ${ }^{2}$, Andrea Corsinotti ${ }^{1}$, Elisa Laurenti ${ }^{1,3}$, Sonia Verp ${ }^{1}$, Sandra Offner ${ }^{1}$, Julien Marquis ${ }^{1}$, Adamandia Kapopoulou ${ }^{1}$, Jiri Vanicek ${ }^{2}$, Didier Trono ${ }^{1}$

$>$ Un adulte en bonne santé produit jusqu'à cent milliards de nouveaux globules rouges chaque jour, afin d'en maintenir les stocks sanguins. Le globule rouge, ou érythrocyte, contient l'hémoglobine responsable de transporter l'oxygène des poumons vers l'ensemble du corps. L'érythrocyte a son origine dans la moelle osseuse, dérivant de la cellule souche hématopoïétique via un processus complexe de prolifération et de différenciation [1, 2]. II reste encore beaucoup à apprendre sur les mécanismes moléculaires de ces événements, non seulement pour comprendre la cause de certains troubles hématologiques, mais aussi pour envisager un jour la production de globules rouges en laboratoire - un enjeu important pour la transfusion.

\section{Le système KRAB/KAP1}

Les génomes des vertébrés supérieurs codent pour des centaines de protéines à doigt de zinc dotées d'un domaine KRAB (KRAB-ZFP). Ces protéines reconnaissent des séquences spécifiques de I'ADN via leurs doigts de zinc, et y recrutent leur cofacteur universel KAPl via le motif KRAB (Krueppel-associated box). KAPl, également connu sous les noms de TRIM28 (protéine à motif tripartite 28), TIFl $\beta$ (facteur de transcription intermédiaire $1 \beta$ ), ou Krip-1 (KRAB-interacting protein 1), agit ensuite comme une plate-forme pour un complexe multimoléculaire qui réprime la transcription par l'induction d'hétérochromatine [3, 4]. Le système KRAB/KAPl a probablement d'abord évolué pour minimiser les
${ }^{1}$ School of Life Sciences and Frontiers in Genetics Program ;

${ }^{2}$ School of Basic Sciences, École

Polytechnique Fédérale de Lausanne (EPFL), 1015 Lausanne, Suisse.

${ }^{3}$ Adresse actuelle: Campbell Family Institute for Cancer Research, Ontario Cancer Institute, Princess Margaret Cancer Centre, University Health Network and Department of Molecular Genetics, University of Toronto, Toronto, Ontario, Canada.

didier.trono@epfl.ch

perturbations du génome induites par I'invasion des rétroéléments [5], mais des données récentes indiquent qu’il contrôle également de multiples aspects de la physiologie des mammifères $[6,7]$.

\section{Défaut de mitophagie en l'absence de KAP1}

Afin d'étudier le rôle potentiel de ce système dans l'hématopoïèse, nous avons construit une souris adulte dont le gène codant pour KAPl est invalidé sélectivement (knock-out, KO) dans les lignées hématopoïétiques. Le phénotype de ces souris se caractérise par une série d'anomalies hématologiques dont la plus remarquable est une anémie due à un arrêt de différenciation des précurseurs des érythrocytes - les 